\title{
HYPERURICEMIA ON ADMISSION PREDICTS SHORT-TERM MORTALITY DUE TO MYOCARDIAL INFARCTION IN A POPULATION WITH HIGH PREVALENCE OF CARDIOVASCULAR RISK FACTORS
}

\author{
Mauricio Mora-Ramírez ${ }^{1}$, Irving O. Estevez-Garcia ${ }^{1}$, María E. Irigoyen-Camacho ${ }^{2}$, Rafael Bojalil ${ }^{1,2}$, \\ Héctor Gonzalez-PACHeCO ${ }^{3}$ AND LUIS M. AmezCuA-Guerra ${ }^{1,2,4 *}$ \\ ${ }^{1}$ Department of Immunology, Instituto Nacional de Cardiología Ignacio Chávez; ${ }^{2}$ Department of Health Care, \\ Universidad Autónoma Metropolitana-Xochimilco; ${ }^{3}$ Coronary Care Unit, Instituto Nacional de Cardiología Ignacio Chávez; \\ ${ }^{4}$ La Salle University, School of Medicine. Mexico City, Mexico
}

\begin{abstract}
Background: National health surveys have revealed an outstandingly high prevalence of obesity, hypertension, and diabetes in Mexico. Objective: To assess whether serum uric acid levels on admission may predict short-term mortality in patients with ST-segment elevation myocardial infarction in a population with an unusually high prevalence of classic cardiovascular risks. Methods: A total of 795 ST-segment elevation myocardial infarction patients undergoing primary reperfusion therapy were classified as having normouricemia or hyperuricemia according to serum uric acid levels at admission, and the occurrence of mortality and major adverse cardiovascular events during coronary care unit stay was assessed. Results: Patients with hyperuricemia ( $n=291$; mean age $61.2 \pm 11.9$ years; $74.8 \%$ males) were older, obese, hypertensive, and had a higher Killip class at admission than those with normouricemia ( $n=504$; mean age $57.6 \pm 11.3$ years; $88.9 \%$ males). Mortality rates were 1.7 and 0.7 cases/100 patients per day of coronary care unit stay in hyperuricemic and normouricemic patients, respectively. Comparatively, no association was observed for the occurrence of major adverse cardiovascular events. After multivariate adjustments, independent predictors for short-term mortality were only Killip class $\geq 2$ (HR: 13.15; 95\% Cl: 5.29-29.85; $p<0.0001$ ) and elevated serum uric acid levels (HR: $1.99 ; 95 \% \mathrm{Cl}: 1.08-3.66 ; \mathrm{p}=0.026$ ). Conclusions: Hyperuricemia on admission remains associated with short-term mortality in ST-segment elevation myocardial infarction patients from a population with an unusually high prevalence of cardiovascular risk factors.
\end{abstract}

Key words: Uric acid. Mortality. Myocardial infarction. Cardiovascular risk factor.

Corresponding author:

*Luis M. Amezcua-Guerra

Department of Immunology

Instituto Nacional de Cardiología Ignacio Chávez

Juan Badiano, 1

Col. Belisario Domínguez, Sección XVI, Del. Tlalpan

C.P. 14080, Ciudad de México, México

Received for publication: 08-02-2017

E-mail: Imamezcuag@gmail.com

Accepted for publication: 12-04-2017

doi: $10.24875 / R I C .17002167$ 


\section{INTRODUCTION}

Coronary artery disease has become the leading cause of morbidity and mortality in the adult population around the world ${ }^{1}$. This may be attributed to an alarming increase in the prevalence of chronic diseases such as obesity, diabetes, hypertension, dyslipidemia, and hyperuricemia. In 2012, the National Health Survey in Mexico (ENSANUT 2012) revealed unusually high figures for these conditions ${ }^{2}$. In fact, 7/10 adult Mexicans were overweight or obese, 3/10 were hypertensive, and $1 / 10$ were diabetic ${ }^{3-5}$. In addition, the Mexico City Diabetes Study, a prospective population-based cohort that included 1,173 participants, reported a prevalence of 26.5 and $19.5 \%$ for asymptomatic hyperuricemia in men and women, respectively ${ }^{6}$. Furthermore, this condition was an undiagnosed characteristic in the majority of these individuals. As a result, these data establish the Mexican population as one with outstandingly high cardiovascular risk and particularly different within the world populations ${ }^{7}$.

Uric acid is the end product of purine metabolism in humans, and increasing evidence suggests that persistently elevated levels of serum uric acid (sUA) increase oxidative stress and ultimately contribute to atherosclerosis ${ }^{8}$. Moreover, elevated sUA levels have been associated with an increase in mortality in a number of cardiovascular diseases including heart failure, hypertension, diabetes, and acute coronary syndromes (ACS) ${ }^{9}$. This serum marker has been studied in patients with ST-segment elevation myocardial infarction (STEMI), reporting an association between sUA concentration at admission and mortality in the short and long terms ${ }^{10-12}$.

Therefore, it is important to ascertain whether sUA levels remain as a reliable prognostic biomarker for short-term mortality in patients with STEMI undergoing reperfusion therapy, considering the unusually high prevalence of additional cardiovascular risks found in the Mexican population.

\section{MATERIALS AND METHODS}

\section{Study design and patients}

The present study was performed in the Coronary Care Unit (CCU) registry data at the Instituto Nacional de Cardiología Ignacio Chávez, Mexico City, Mexico.
Briefly, this is a comprehensive database that processes data from all consecutive adult patients admitted to the CCU from January 2006 onwards. It includes demographic data and clinical information, medical procedures, coronary angiography characteristics, and subsequent in-hospital event occurrence. The data are periodically integrated, reviewed, and checked for accuracy. As of January 2012, sUA levels began to be measured and systematically recorded in all patients at admission.

For this longitudinal and observational study, data from all individuals admitted between January 2012 and December 2014 were thoroughly examined. We included patients who met the following criteria: (i) diagnosis of STEMI based on clinical features, electrocardiographic changes, and biochemical markers of cardiac necrosis according to the 2001 American College of Cardiology definitions ${ }^{13}$; (ii) had sUA measurement on admission; and (iii) underwent myocardial reperfusion therapy (thrombolytic therapy or primary percutaneous coronary intervention $[\mathrm{PCI}]$ ) within 12 hours of onset. After that, we excluded patients with: (i) current use of uric acid-lowering drugs (e.g. allopurinol, probenecid, benzbromarone) or thiazides; (ii) active neoplastic disease; (iii) end-stage renal disease with dialysis; (iv) history of gouty arthritis or urolithiasis; and (v) missing values in the data registry.

\section{Outcomes}

The primary outcome of this study was mortality by any cause during CCU stay. In addition, the occurrence of cardiogenic shock, re-infarction, fatal arrhythmias, acute pulmonary edema, acute heart failure and stroke during CCU stay was registered and analyzed as an unique composite outcome, namely major adverse cardiovascular events (MACE) ${ }^{14}$.

\section{Laboratory procedures}

Measurement of sUA levels on admission was performed by spectrophotometry (Hitachi 902; Roche Diagnostics, Indianapolis, IN, USA) and the results were expressed in milligrams per deciliter. The cutoff value for the levels of sUA to classify a patient as having hyperuricemia was $\geq 7 \mathrm{mg} / \mathrm{dl}$ in men and $\geq 6 \mathrm{mg} / \mathrm{dl}$ in women, as previously indicated in the National Health and Nutrition Examination Survey (NHANES) ${ }^{15}$.

This study was conducted in accordance with the principles of the Declaration of Helsinki and approved 
by the local ethics committee (Comisión de Bioética del Instituto Nacional de Cardiología Ignacio Chávez). An informed consent was obtained from all patients.

\section{Statistical analysis}

Data distribution was assessed using the KolmogorovSmirnov test. Continuous variables were presented as mean \pm standard deviation (SD), while categorical variables were described as percentages and proportions. To assess differences, Student's $t$ tests or $\chi^{2}$ tests were used as appropriate. The cumulative survival curves for cardiovascular mortality during CCU stay were constructed using the Kaplan-Meier method, with differences assessed using the log-rank (Mantel-Cox) test, and the hazard ratio (HR) with $95 \%$ confidence interval $(95 \% \mathrm{Cl})$ was obtained using a Cox proportional hazards model. The primary outcome was the occurrence of death. The length of survival was defined as the time of entry into the study to the occurrence of death or discharge from the CCU. Discharged patients were considered as censored observations at the time of their last clinical evaluation at the CCU. Multivariate models by Cox regression were performed to evaluate the role of different variables on mortality, and the $\mathrm{HR}$ with $95 \% \mathrm{Cl}$ was used to describe associations. All analyses were two-tailed and a $p$ value of $\leq 0.05$ was set for significance. The GraphPad Prism v7.00 (GraphPad Software, San Diego CA, USA) and STATA v12 (STATA Corp LP, College Station, Texas, USA) statistical software were used for data analysis.

\section{RESULTS}

A total of 866 patients with STEMI who underwent reperfusion therapy were admitted to the CCU during the study period. Of these, 71 patients ( $8.19 \%$ ) were excluded from the analysis because of the concurrent use of thiazides (39 patients) or uric acid-lowering drugs (18), history of gouty arthritis (6) or urolithiasis (2), chronic kidney disease in renal replacement therapy (2), or missing data (2). No differences were observed between included and excluded patients in terms of the main clinical and laboratory characteristics at admission (data not shown).

A total of 795 patients were finally included in the analysis and their main demographic and clinical data are summarized in table 1. As noted, 291 (36.6\%) patients were classified as having hyperuricemia and $504(63.4 \%)$ as having normouricemia. Patients with hyperuricemia were generally more obese ( 25.4 vs. $17.6 \% ; p=0.03)$, older ( $61.2 \pm 11.9$ vs. $57.6 \pm 11.3$; $p<0.0001$ ), hypertensive ( 54.0 vs. $40.8 \%$; $<0.0001$ ), and female ( 24 vs. $11 \%$; $<<0.0001$ ) than their counterparts with normouricemia.

Clinical severity was assessed using the Killip system and a class $\geq 2$ was found in $45.0 \%$ of patients with hyperuricemia, compared to $25.1 \%$ in cases with normouricemia $(p<0.0001)$. Notably, thrombolysis was the most frequently indicated myocardial reperfusion therapy in patients with normouricemia ( $43.8 \mathrm{vs.}$ $35.7 \% ; p=0.03$ ), whereas primary $\mathrm{PCl}$ was the most used in patients with hyperuricemia ( 64.3 vs. $56.2 \%$; $p=0.03$ ). Other baseline characteristics of patients are summarized in table 1 .

With regard to clinical outcomes, the length of stay at the CCU and the occurrence of MACE were similar in both groups (Table 2). However, the proportion of patients dying at the CCU was increased in patients with elevated sUA levels at admission ( 10.3 vs. $3.9 \%$ ), and the mortality rates were 1.7 cases $/ 100$ patients per day of CCU stay in patients with hyperuricemia and 0.7 cases/100 patients per day of CCU stay in those with normouricemia. In parallel, hyperuricemia conferred a HR of 2.13 ( $95 \% \mathrm{Cl}: 1.26-3.98 ; \mathrm{p}=0.005$ ) for mortality (Fig. 1).

Table 3 summarizes the main clinical data on admission of patients who lived and those who died during follow-up. A significantly higher frequency of diabetes and hyperuricemia was observed, as well as a higher Killip score on admission in patients who eventually died during follow-up. Table 4 summarizes univariate and multivariate predictors of mortality. As noted, multivariate analyses showed that Killip class $\geq 2$ (HR: 13.15; 95\% Cl: 5.29-29.85; $\mathrm{p}<0.0001$ ), and high levels of sUA (HR: $1.99 ; 95 \% \mathrm{Cl}: 1.08-3.66 ; \mathrm{p}=0.026$ ) remained as independent predictors of mortality during CCU stay in patients with STEMI.

\section{DISCUSSION}

In this longitudinal study of 795 patients with STEMI who underwent early myocardial reperfusion therapy, 
Table 1. Baseline characteristics of patients classified according to serum uric acid levels

\begin{tabular}{|c|c|c|c|c|}
\hline & Total & $\begin{array}{l}\text { Patients with } \\
\text { hyperuricemia } \\
(n=291)\end{array}$ & $\begin{array}{l}\text { Patients with } \\
\text { normouricemia } \\
\quad(n=504)\end{array}$ & $P$ value \\
\hline Age, years & $59.0 \pm 11.7$ & $61.2 \pm 11.9$ & $57.6 \pm 11.3$ & $<0.0001$ \\
\hline Men, n (\%) & $668(83.0)$ & $220(74.8)$ & $448(88.9)$ & $<0.0001$ \\
\hline BMI, kg/m² & $27.3 \pm 4.2$ & $27.8 \pm 4.6$ & $27.1 \pm 4.0$ & 0.03 \\
\hline $\mathrm{BMI}>30 \mathrm{~kg} / \mathrm{m}^{2}, \mathrm{n}(\%)$ & $163(20.5)$ & $74(25.4)$ & $89(17.6)$ & 0.01 \\
\hline Active smoker, n (\%) & $460(57.8)$ & $156(53.0)$ & $304(60.3)$ & 0.05 \\
\hline Hypertension, n (\%) & $364(45.7)$ & $158(54.0)$ & $206(40.8)$ & $<0.0001$ \\
\hline Dyslipidemia, n (\%) & $232(29.1)$ & $85(29.2)$ & $147(29.2)$ & 0.9 \\
\hline Diabetes, n (\%) & $310(38.9)$ & $115(39.5)$ & $195(38.6)$ & 0.7 \\
\hline Previous ACS, n (\%) & $110(13.8)$ & $36(12.3)$ & $74(14.6)$ & 0.2 \\
\hline Uric acid, mg/dl & $6.4 \pm 1.9$ & $8.3 \pm 1.6$ & $5.3 \pm 1.1$ & $<0.0001$ \\
\hline Killip class $\geq 2$, n (\%) & $258(32.5)$ & $131(45.0)$ & $127(25.1)$ & $<0.0001$ \\
\hline Thrombolysis, n (\%) & $325(40.8)$ & $104(35.7)$ & $221(43.8)$ & 0.03 \\
\hline Primary $\mathrm{PCl}, \mathrm{n}(\%)$ & $470(59.0)$ & $187(64.3)$ & $283(56.1)$ & 0.03 \\
\hline ASA, n (\%) & $789(99.2)$ & $288(98.9)$ & $501(99.4)$ & 0.1 \\
\hline Clopidogrel, n (\%) & $665(83.0)$ & $245(84.1)$ & $420(83.3)$ & 0.8 \\
\hline Unfract heparin, $\mathrm{n}(\%)$ & $577(72.0)$ & $222(76.3)$ & $355(70.4)$ & 0.08 \\
\hline LMWH, n (\%) & $253(31.0)$ & $85(29.0)$ & $168(33.3)$ & 0.2 \\
\hline Statins, n (\%) & $791(99.4)$ & $288(98.9)$ & $503(99.8)$ & 0.2 \\
\hline
\end{tabular}

Data are presented as mean \pm standard deviation unless otherwise specified. Significant $p$ values are shown in bold.

ACS: acute coronary syndrome; ASA: acetylsalicylic acid; BMI: body mass index; LMWM: low-molecular-weight heparin; PCI: percutaneous coronary intervention.

Table 2. Main clinical outcomes in patients with ST-segment elevation myocardial infarction during their coronary care unit stay

\begin{tabular}{|c|c|c|c|c|}
\hline & Total & $\begin{array}{l}\text { Patients with } \\
\text { hyperuricemia } \\
(n=291)\end{array}$ & $\begin{array}{l}\text { Patients with } \\
\text { normouricemia } \\
(n=504)\end{array}$ & $P$ value \\
\hline Length of stay, days & $5.6 \pm 4.0$ & $5.9 \pm 4.4$ & $5.4 \pm 3.8$ & 0.09 \\
\hline CCU mortality, n (\%) & $50(6.5)$ & $30(10.3)$ & $20(3.9)$ & 0.0007 \\
\hline Re-infarction, n (\%) & $9(1.1)$ & $2(0.6)$ & $7(1.3)$ & 0.4 \\
\hline Acute cardiac failure, n (\%) & $8(1.0)$ & $4(1.3)$ & $4(0.8)$ & 0.4 \\
\hline Acute pulmonary edema, $\mathrm{n}(\%)$ & $9(1.1)$ & $6(2.0)$ & $3(0.5)$ & 0.08 \\
\hline Cardiogenic shock, n (\%) & $24(3.0)$ & $13(4.5)$ & $11(2.1)$ & 0.08 \\
\hline Stroke, n (\%) & $5(0.6)$ & $1(0.3)$ & $4(0.7)$ & 0.6 \\
\hline MACE, n (\%) & $55(6.9)$ & $26(8.9)$ & $29(5.7)$ & 0.1 \\
\hline
\end{tabular}

Data are presented as mean \pm standard deviation unless otherwise specified. $\mathrm{P}$ data are presented as mean \pm standard deviation unless otherwise specified, and significant values are shown in bold.

CCU: coronary care unit; MACE: major adverse cardiovascular events.

it was found that elevated sUA levels at admission might independently predict the short-term mortality in a group of individuals with a high prevalence of classical cardiovascular risk factors.

The concentration of uric acid reflects the balance of purine metabolism by xanthine oxidase (XO) in the liver and its elimination by the kidneys ${ }^{8}$. Therefore, levels of sUA are closely related to the enzymatic activity of $\mathrm{XO}$, which is directly responsible for tissue oxidative stress ${ }^{16}$. In fact, $X O$ activity is an important source of reactive oxygen species (ROS), and elevated levels of sUA further contribute to a downregulation of extracellular superoxide dismutase activity, the main antioxidant system against $\mathrm{ROS}^{17,18}$. A pro-oxidative environment is 
Figure 1. Survival estimates for patients with ST-segment elevation myocardial infarction. Kaplan-Meier survival plot of patients according to serum uric acid levels at hospital admission. Overall survival in patients with hyperuricemia decreased significantly after 30 days of follow-up.

CCU: coronary care unit.

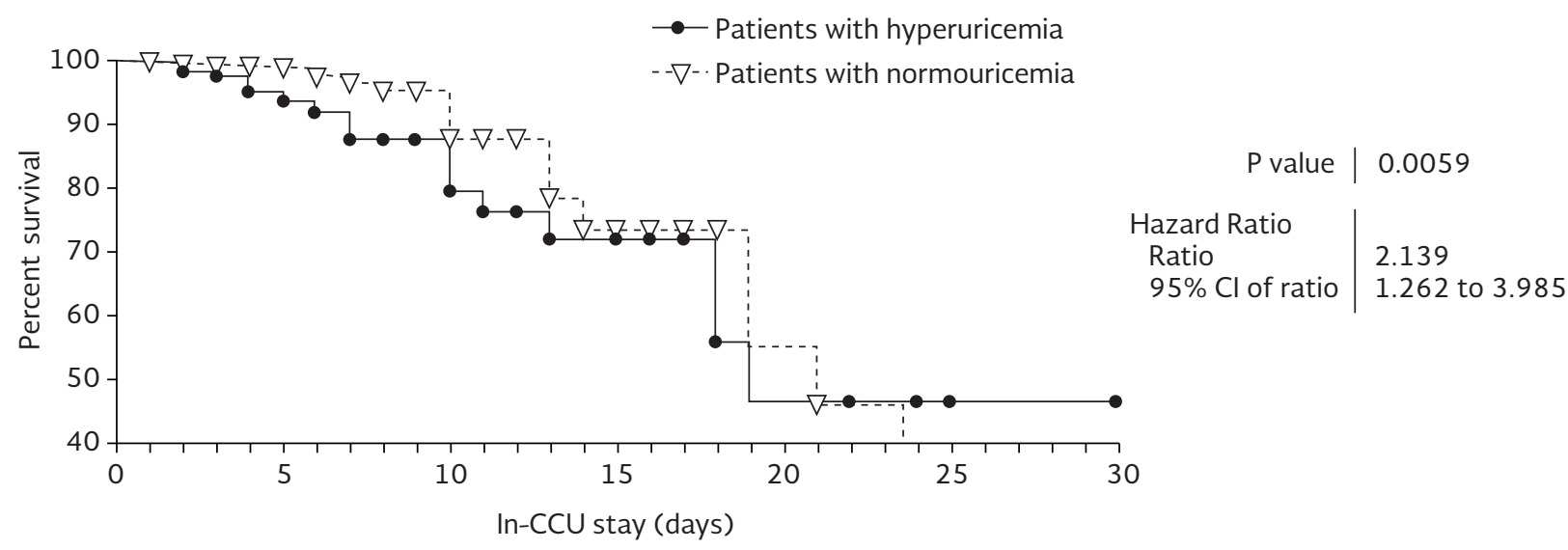

Table 3. Comparative information at the time of admission to the coronary care unit between living and deceased patients with ST-segment elevation myocardial infarction

\begin{tabular}{lccc}
\hline & $\begin{array}{c}\text { Alive } \\
(\mathrm{n}=\mathbf{7 4 5})\end{array}$ & $\begin{array}{c}\text { Dead } \\
(\mathrm{n}=\mathbf{5 0})\end{array}$ & $\boldsymbol{P}$ value \\
\hline Age, years & $58.7 \pm 11.6$ & $62.0 \pm 10.9$ & 0.054 \\
Men, $\mathrm{n}(\%)$ & $626(84.0)$ & $42(84.0)$ & 0.9 \\
BMl, $\mathrm{kg} / \mathrm{m}^{2}$ & $27.3 \pm 4.2$ & $27.5 \pm 4.6$ & 0.7 \\
Hypertension, $\mathrm{n}(\%)$ & $339(45.5)$ & $25(50.0)$ & 0.5 \\
Dyslipidemia, $\mathrm{n}(\%)$ & $222(29.7)$ & $10(20.0)$ & 0.15 \\
Diabetes, $\mathrm{n}(\%)$ & $283(37.9)$ & $27(54.0)$ & 0.03 \\
Killip class $\geq 2, \mathrm{n}(\%)$ & $215(28.8)$ & $43(86.0)$ & $<0.0001$ \\
Serum uric acid, mg/dl & $6.3 \pm 1.8$ & $8.2 \pm 3.1$ & $<0.0001$ \\
Thrombolysis, $\mathrm{n}(\%)$ & $310(41.6)$ & $35(30.0)$ & 0.13 \\
Primary PCl, $\mathrm{n}(\%)$ & $435(58.4)$ & $35(70.0)$ & 0.13 \\
\hline
\end{tabular}

Data are presented as mean \pm standard deviation unless otherwise specified. Significant $\mathrm{p}$ values are shown in bold. $\mathrm{BMI}$ : body mass index; $\mathrm{PCl}$ : percutaneous coronary intervention.

Table 4. Crude and adjusted hazard ratios for mortality in patients with ST-segment elevation myocardial infarction

\begin{tabular}{|c|c|c|c|c|c|c|}
\hline & \multicolumn{3}{|c|}{ Crude } & \multicolumn{3}{|c|}{ Adjusted* } \\
\hline & HR & $(95 \% \mathrm{Cl})$ & $P$ value & HR & $(95 \% \mathrm{Cl})$ & $P$ value \\
\hline Age, years & 1.01 & $(1.01-1.04)$ & 0.047 & 1.01 & $(0.90-1.03)$ & 0.47 \\
\hline Sex (male) & 0.80 & $(0.40-1.61)$ & 0.54 & 0.30 & $(0.10-1.30)$ & 0.12 \\
\hline BMI kg/m² & 1.02 & $(0.95-1.07)$ & 0.67 & 1.02 & $(0.95-1.09)$ & 0.53 \\
\hline Hypertension & 1.28 & $(0.74-2.20)$ & 0.50 & 0.99 & $(0.52-1.70)$ & 0.84 \\
\hline Diabetes & 2.01 & $(1.16-3.46)$ & 0.012 & 1.50 & $(0.86-2.70)$ & 0.14 \\
\hline Killip class $\geq 2$ & 12.80 & $(5.70-25.99)$ & $<0.0001$ & 13.15 & $(5.29-29.85)$ & $<0.0001$ \\
\hline Hyperuricemia & 2.83 & $(1.62-4.90)$ & $<0.0001$ & 1.99 & $(1.08-3.66)$ & 0.026 \\
\hline Previous ACS & 1.69 & $(0.87-3.29)$ & 0.10 & 1.80 & $(0.90-3.70)$ & 0.08 \\
\hline Thrombolysis & 0.59 & $(0.32-1.08)$ & 0.08 & 0.67 & $0.07-5.70)$ & 0.65 \\
\hline Primary $\mathrm{PCl}$ & 1.46 & $(0.81-2.60)$ & 0.10 & 0.75 & $(0.90-6.10)$ & 0.79 \\
\hline
\end{tabular}

Significant $\mathrm{p}$ values are shown in bold. *Adjusted by variables listed in the table.

HR: hazard ratio; $95 \% \mathrm{Cl}$, confidence intervals; BMI, body mass index; ACS, acute coronary syndrome; PCl, percutaneous coronary intervention. 
created, which favors a decrease in the production of nitric oxide by endothelial cells, and this in turn inhibits vasodilation and promotes inflammation and endothelial dysfunction ${ }^{19,20}$. This provides a plausible pathophysiological link between steady hyperuricemia and chronic oxidative stress that contributes to atherosclerotic disease ${ }^{21}$. However, recent evidence suggests that uric acid may play a role as alarmin in several inflammatory pathways mediated by pattern recognition receptors. In particular, uric acid may activate the nucleotide-binding oligomerization domainlike receptors, a subset of intracellular sensors for damage-associated molecular patterns that ultimately activate inflammasomes, multi-molecular complexes that serve as platforms for the activation of mature forms of interleukin-1 $\beta$ and interleukin-18. This appears to be relevant in conditions characterized by extensive cell necrosis, such as myocardial infarction, suggesting that elevated levels of sUA may also be a reflection of the extent of myocardial tissue damage ${ }^{22,23}$.

Several studies have established an association between SUA levels and coronary artery disease, which results in the acceptance of hyperuricemia as a risk factor for several cardiovascular diseases such as chronic heart failure and pulmonary hypertension ${ }^{24,25}$. Hyperuricemia on admission has been previously recognized as independently associated with mortality in patients with ACS; nonetheless, controversy still exists on whether elevated sUA levels are just a simple risk marker (due to its close association to other cardiovascular risk factors) or is truly an independent risk factor for mortality ${ }^{26}$. In this line, Kojima, et al. demonstrated that elevated sUA predicts the future development of congestive heart failure and mortality in Japanese patients with ACS ${ }^{27}$. A recent study from Turkey showed that patients with hyperuricemia and STEMI who underwent $\mathrm{PCl}$ had increased inhospital mortality compared to those with normouricemia (Odds ratio [OR]: $2.03 ; 95 \% \mathrm{Cl}: 1.25-3.75$; $\mathrm{p}=0.006)^{12}$. In addition, Ndrepepa, et al. studied German patients with ACS and found that higher sUA levels were associated with higher mortality rates in a one-year follow-up period (OR 3.56 ; $95 \% \mathrm{Cl}$ : $2.80-$ 4.52; $p<0.001$ ), although the maximum impact of hyperuricemia over mortality occurred within the first seven days of follow-up ${ }^{11}$. In contrast, Lazzeri, et al. recently described 856 STEMI patients admitted to the $\mathrm{CCU}$ after $\mathrm{PCl}$, and concluded that elevated sUA levels predict the occurrence of complications during CCU stay, but not early mortality, after adjustment for renal function and extent of myocardial necrosis evaluated by the Killip class ${ }^{26}$.

The Mexican population is of great interest due to an alarmingly high prevalence of classic cardiovascular risk factors. In the last 12 years, overweight and obesity have reached $71.3 \%$ in this particular adult population, an increase of almost 10\% compared with the year $2000(61.9 \%$ in $2000,69.7 \%$ in 2006 , and $71.3 \%$ in 2012$)^{3}$. In addition, the prevalence of diabetes increased from 4.6 to $9.2 \%$ in the same time period (2.1 million individuals in 2000, 3.7 million in 2006, and 6.4 million in 2012) $)^{5}$. In regards to hypertension, this condition currently affects up to $31.5 \%$ of the Mexican population, remaining mostly unchanged in the last decade $(30.1 \%$ in $2000,31.6 \%$ in 2006 , and $31.5 \%$ in 2012$)^{4}$. The present study has demonstrated that elevated sUA levels at admission are associated with higher CCU mortality in patients with STEMI who underwent early myocardial reperfusion therapy, in a population with an unusually high prevalence of cardiovascular risk factors. Moreover, hyperuricemia proved to be an independent risk factor for CCU mortality when adjusting by multiple variables.

We are aware that our study has limitations, mainly the lack of follow-up of the patients after discharge from the CCU, so that drawing conclusions from the long-term outcomes was not possible. Although the patients were prospectively included, data analyses were done retrospectively. In addition, inflammatory and oxidative stress markers were not measured. Finally, the use of uric acid-lowering drugs after the identification of hyperuricemia in patients with STEMI should still be evaluated for its potential therapeutic effect on the long-term follow-up.

In conclusion, elevated sUA levels at admission identify a subset of patients with a high mortality risk during their CCU stay, independently of other risk factors, including the extent of myocardial dysfunction. Hyperuricemia remained an independent predictor of mortality in a population with an unusually high prevalence of cardiovascular risk factors, thus describing an easily available laboratory measurement that may further assess the increased risk of mortality in an already afflicted population. 


\section{REFERENCES}

1. Kolansky DM. Acute coronary syndromes: morbidity, mortality, and pharmacoeconomic burden. Am J Manag Care. 2009; 15(Suppl):S36-41.

2. Gutiérrez J, Rivera-Dommarco J, Shamah-Levy T, et al. Encuesta Nacional de Salud y Nutrición 2012. Resultados Nacionales. Salud Publica Mex. 2012;196.

3. Barquera S, Campos-Nonato I, Hernández-Barrera L, Pedroza A, Rivera-Dommarco JA. [Prevalence of obesity in Mexican adults 2000-2012]. Salud Publica Mex. 2013;55(Suppl 2): S151-60.

4. Campos-Nonato I, Hernández-Barrera L, Rojas-Martínez R, Pedroza A, Medina-García C, Barquera-Cervera S. [Hypertension: prevalence, early diagnosis, control and trends in Mexican adults]. Salud Publica Mex. 2013;55(Suppl 2):S144-50.

5. Jiménez-Corona A, Aguilar-Salinas CA, Rojas-Martínez R, Hernández-Ávila M. [Type 2 diabetes and frequency of prevention and control measures]. Salud Publica Mex. 2013;55(Suppl 2): S137-43.

6. López-Molina R, Parra-Cabrera S, López-Ridaura R, GonzálezVillalpando ME, Ferrannini E, González-Villalpando C. Sweetened beverages intake, hyperuricemia and metabolic syndrome: the Mexico City Diabetes Study. Salud Publica Mex. 2013;55: 557-63.

7. WHO. Global Health Risks: Mortality and burden of disease attributable to selected major risks. Bull World Health Organ. 2009;87:646.

8. Puddu P, Puddu GM, Cravero E, Vizioli L, Muscari A. The relationships among hyperuricemia, endothelial dysfunction, and cardiovascular diseases: Molecular mechanisms and clinical implications. J Cardiol. 2012;59:235-42.

9. Culleton BF, Larson MG, Kannel WB, Levy D. Serum uric acid and risk for cardiovascular disease and death: the Framingham Heart Study. Ann Intern Med. 1999;131:7-13.

10. Lazzeri C, Valente S, Chiostri M, Sori A, Bernardo P, Gensini GF. Uric acid in the acute phase of ST elevation myocardial infarction submitted to primary $\mathrm{PCl}$ : its prognostic role and relation with inflammatory markers: a single center experience. Int J Cardiol. 2010;138:206-9.

11. Ndrepepa G, Braun $\mathrm{S}$, Haase $\mathrm{H}-\mathrm{U}$, et al. Prognostic value of uric acid in patients with acute coronary syndromes. Am J Cardiol. 2012;109:1260-5

12. Kaya MG, Uyarel $H$, Akpek $M$, et al. Prognostic value of uric acid in patients with ST-elevated myocardial infarction undergoing primary coronary intervention. Am J Cardiol. 2012;109: 486-91.
13. Cannon CP, Battler A, Brindis RG, et al. American College of Cardiology key data elements and definitions for measuring the clinical management and outcomes of patients with acute coronary syndromes. J Am Coll Cardiol. 2001;38:2114-30.

14. Williams DO, Abbott JD, Kip KE; DEScover Investigators. Outcomes of 6906 patients undergoing percutaneous coronary intervention in the era of drug-eluting stents: Report of the DEScover Registry. Circulation. 2006;114:2154-62.

15. Zhu Y, Pandya BJ, Choi HK. Prevalence of gout and hyperuricemia in the US general population: The National Health and Nutrition Examination Survey 2007-2008. Arthritis Rheum. 2011;63: 3136-41.

16. Stocker R, Keaney JF. New insights on oxidative stress in the artery wall. J Thromb Haemost. 2005;3:1825-34.

17. Alcaino H, Greig D, Chiong $M$, et al. Serum uric acid correlates with extracellular superoxide dismutase activity in patients with chronic heart failure. Eur J Heart Fail. 2008;10:646-51.

18. George J, Struthers AD. Role of urate, xanthine oxidase and the effects of allopurinol in vascular oxidative stress. Vasc Health Risk Manag. 2009;5:265-72.

19. Godber BL, Doel JJ, Sapkota GP, et al. Reduction of nitrite to nitric oxide catalyzed by xanthine oxidoreductase. J Biol Chem. 2000;275:7757-63.

20. Meneshian A, Bulkley GB. The physiology of endothelial xanthine oxidase: From urate catabolism to reperfusion injury to inflammatory signal transduction. Microcirculation. 2002;9:161-75.

21. Krishnan E, Pandya BJ, Chung L, Dabbous O. Hyperuricemia and the risk for subclinical coronary atherosclerosis - data from a prospective observational cohort study. Arthritis Res Ther. 2011; 13:R66.

22. Ghaemi-Oskouie F, Shi Y. The role of uric acid as an endogenous danger signal in immunity and inflammation. Curr Rheumatol Rep. 2011;13:160-6

23. Matzinger P. Tolerance, danger, and the extended family. Annu Rev Immunol. 1994:12:991-1045.

24. Bergamini C, Cicoira M, Rossi A, Vassanelli C. Oxidative stress and hyperuricaemia: pathophysiology, clinical relevance, and therapeutic implications in chronic heart failure. Eur J Heart Fail. 2009;11:444-52.

25. Nagaya N, Uematsu M, Satoh T, et al. Serum uric acid levels correlate with the severity and the mortality of primary pulmonary hypertension. Am J Respir Crit Care Med. 1999;160:487-92.

26. Lazzeri C, Valente S, Chiostri M, Picariello C, Gensini GF. Uric acid in the early risk stratification of ST-elevation myocardial infarction. Intern Emerg Med. 2012;7:33-9.

27. Kojima S, Sakamoto T, Ishihara $M$, et al. Prognostic usefulness of serum uric acid after acute myocardial infarction (The Japanese Acute Coronary Syndrome Study). Am J Cardiol. 2005;96:489-95. 